\title{
Modelo para la integración curricular de ingeniería industrial en Mercosur
}

\author{
Model to integrate industrial engineering curriculum in Mercosur
}

\author{
Diana I. Paravié ${ }^{*} \quad$ Edwin V. C. Galdamez ${ }^{2} \quad$ Gislaine Camila L. Leal ${ }^{2}$ \\ Franco J. Chiodi ${ }^{3}$ Silvia B. Urrutia ${ }^{1}$ Fernando J. Cusolito $^{3}$ \\ Recibido 29 de mayo de 2017, aceptado 21 de enero de 2018 \\ Received: May 29, 2017 Accepted: January 21, 2018
}

\begin{abstract}
RESUMEN
El proceso de cooperación internacional integra las funciones de enseñanza e investigación dando a las universidades una apertura hacia la internacionalización.

Dos países miembros del Mercosur, Argentina y Brasil, señalan en los últimos años esfuerzos gubernamentales para constituir espacios de cooperación universitaria a través de la movilidad docente y estudiantil.

En este contexto, la Ingeniería es una disciplina importantísima para el desarrollo industrial y la innovación productiva. Para impulsarla se necesita robustecer el proceso formativo de alumnos y profesores para lograr un profesional con competencias de desempeño en entornos internacionales.

Para esto dos universidades argentinas y una brasilera establecieron un proceso conjunto de movilidad e integración internacional de la carrera Ingeniería Industrial, con el objetivo principal de presentar el diseño e implementación de un modelo de cooperación internacional para la Integración Curricular de Ingeniería Industrial que permita formar Ingenieros Industriales con características profesionales importantes para el egreso. El modelo es impulsado por un punto de partida, para dar luego inicio a un ciclo iterativo de actividades (representadas por diversas acciones), para lograr un resultado científico y de integración curricular.

Los resultados obtenidos a destacar son intercambio de conocimientos, experiencias en métodos de aprendizaje y actividades culturales e industriales entre otros (ámbito docente); oportunidad de intercambio, discusión de conceptos y herramientas relacionadas con su carrera (ambiente estudiantil) y comparación internacional de los currículos de Ingeniería Industrial (ámbito institucional).

A futuro se plantea el logro de un currículo integrado en Ingeniería Industrial para todas las partes interesadas incluida la industria regional.
\end{abstract}

Palabras clave: Educación superior, universidad, modelo de cooperación internacional, ingeniería, Argentina, Brasil.

\footnotetext{
ABSTRACT

The international cooperation process integrates the teaching and research functions giving universities an openness towards internationalization. In recent years, two Mercosur countries, Argentina and Brazil have pointed out government efforts to create spaces for inter-university cooperation through teaching and student mobility. In this context, Engineering is an relevant discipline for industrial development and

1 Facultad de Ingeniería. Universidad Nacional del Centro de la Provincia de Buenos Aires. Av. Del Valle 5737. Olavarría, Argentina. E-mail: dparavie@fio.unicen.edu.ar; surrutia@fio.unicen.edu.ar

2 Programa de Pós-graduación en Ingeniería de Producción. Universidad Estadual de Maringá. Av. Colombo, 5790. J. Universitário Maringá.PR. Brasil.E-mail: evcgaldamez@uem.br; gclleal@uem.br

3 Instituto de Industria. Universidad Nacional de General Sarmiento. Av. J. M. Gutierrez 1150. Los Polvorines, Argentina. E-mail: fchiodi@ungs.edu.ar; fcusolit@ungs.edu.ar

* Autor de correspondencia
} 
productive innovation. To boost it, it is necessary to strengthen the training process of students and professors to achieve a professional with performance competencies in international environments. For this purpose two Argentine and one Brazilian universities established a joint process of mobility and international integration of the Industrial Engineering career, with the main objective of presenting the design and implementation of an international cooperation model for the Integration of Curricular Industrial Engineering that allows training Industrial Engineers with important professional characteristics for success. The model is driven by a starting point (drivers), to then start an iterative cycle of activities (represented by various actions), to achieve a scientific result and curriculum integration (output). The results obtained are knowledge exchange, experiences in learning methods and cultural and industrial activities (teaching area); the opportunity of exchange, discussion of concepts and tools related to this program (student environment) and international comparison of Industrial Engineering curricula (institutional scope). In the future it is proposed the achievement of an integrated curriculum in Industrial Engineering for all the interested parties including the regional industry.

Keywords: Higher education, university, international cooperation model, engineering, Argentina, Brasil.

\section{INTRODUCCIÓN}

Las instituciones de Educación Superior son piezas fundamentales en el desarrollo de las sociedades. El compromiso entre la Universidad y la Sociedad donde está inserta, hace de la transferencia de conocimientos y la tecnología una misión esencial, con igual jerarquía que la de formar recursos humanos y crear conocimiento. Una Universidad comprometida con la sociedad debe responder a sus demandas globales, regionales y locales, dimensiones que provocan diferentes perspectivas, prácticas profesionales y experiencias sociales [1].

Se destaca que la educación superior demanda una dimensión global, formada a partir de un sistema de conocimientos, flujo de información, redes sociales y el movimiento de las personas en una escala internacional [1].

La internacionalización es un proceso de integrar una dimensión internacional o intercultural a las funciones de enseñanza, investigación, dando a la universidad un sentido de apertura y de interacción internacional, generando mecanismos de cooperación [2].

El intercambio y movilidad interuniversitaria es un valor en sí mismo que permite conocer diversas realidades académicas, económicas y sociales dentro y fuera del país, fortaleciendo y potenciando la formación de los futuros profesionales de áreas de ingeniera, derecho, medicina, sociales, etc. Una de las experiencias más consolidada sobre educación superior global e integración internacional es el Bologna Process, que reúne e integra la educación superior de los países europeos [3].

En los países del Mercosur, se percibe en los últimos años en lo que respecta a los programas de movilidad, esfuerzos tanto gubernamentales como de las instituciones de educación superior en dirigir la mirada hacia la región y constituir espacios de cooperación académica e institucional a través de la movilidad de estudiantes de grado y posgrado, docentes-investigadores y gestores.

"El Estado argentino viene impulsando y acompañando desde el año 2003 los procesos de internacionalización del sistema universitario argentino con el objetivo de insertar a las Instituciones de Educación Superior (IES) en el ámbito regional e internacional con una fuerte impronta de liderazgo en los procesos de integración. En particular, los programas, proyectos $\mathrm{y}$ acciones diseñados e implementados contribuyen a la mejora de la calidad y al incremento de la pertinencia de las ofertas académicas de las IES Argentinas" [4].

Otra iniciativa de intercambio internacional es el "Programa Ciencia sin Fronteras" conducido por Brasil con el objetivo de insertar estudiantes, principalmente, de Ingeniería en sistemas educacionales competitivos de áreas tecnológicas y de innovación, totalizando, más de 100 mil intercambios de 2010 a 2016 [5]. Un proyecto ejecutado a nivel regional es el Sistema de Acreditación Regional de Carreras Universitarias 
de los Estados partes del Mercosur (ARCU-SUR), donde el sistema ofrece garantía pública, entre los países de la región, del nivel académico y científico de las carreras y sus egresados [6].

En este contexto, siendo la Ingeniería una disciplina fundamental para el desarrollo industrial y la innovación productiva, es importante consolidar la formación a través del fortalecimiento y actualización de contenidos, como también inculcar, durante el proceso formativo, competencias, capacidades, actitudes y aptitudes que permitan generar un profesional de alta capacitación técnica con compromiso social, conciencia ambiental y capacidad de liderazgo, en entornos internacionales.

Dentro de este marco, tres universidades del Mercosur, dos de ellas argentinas y la tercera brasilera, han establecido un proceso conjunto de movilidad e integración internacional de la carrera de Ingeniería Industrial.

Las instituciones de educación superior participantes en este proceso son la Universidad Nacional de General Sarmiento (UNGS-ARG), la Universidad Nacional del Centro de la Provincia de Buenos Aires (UNICEN-ARG) y la Universidad Estadual de Maringá (UEM-BR).

En este contexto, el objetivo principal de este trabajo consiste en presentar el diseño e implementación de un modelo de cooperación internacional para la Integración Curricular de Ingeniería Industrial, $\mathrm{I}^{2} \mathrm{EC}$ (Integration of the Industrial Engineering Curriculum).

La integración curricular de Ingeniería Industrial de dos importantes países de América del Sur representa la oportunidad de establecer un padrón educacional. Un padrón de disciplinas técnicas, tecnológicas, gerenciales y humanísticas permite con que profesionales formados en diferentes países actúen en industrias regionales, creando un ambiente favorable para la competitividad, innovación e mejora de la calidad de productos y servicios.

Una iniciativa a nivel nacional en Argentina es el Sistema Nacional de Reconocimiento Académico, que permite contar con un sistema articulado a través de mutuos reconocimientos de tramos curriculares, ciclos, prácticas, asignaturas u otras experiencias formativas [7].

Una educación más amplia en la Ingeniería Industrial genera habilidades y competencias relacionadas con liderazgo, comunicación oral y escrita, emprendedorismo, dominio de más un idioma, entre otros aspectos. Elementos que potencializan un uso más eficiente de los recursos humanos, tecnológicos y económicos de industrias.

Las directrices propuestas permiten crear una referencia para proyectos de cooperación internacional de similares características. Este trabajo también reduce el gap de la literatura ya que existe una escasez de trabajos dentro de esta temática, demostrando que el tema no recibe la debida atención en esta área [8].

En la primera sección se exponen los principales conceptos teóricos que guían el desarrollo del trabajo, y el marco normativo que contextualiza a la carrera de Ingeniería Industrial en cada país. A continuación se presenta y explica el modelo propuesto, denominado $\mathrm{I}^{2} \mathrm{EC}$, para llevar adelante la ejecución de la integración curricular. La tercera sección comenta las acciones ejecutadas para implementar el modelo entre estas tres universidades. Finalmente se sintetizan las conclusiones alcanzadas, así como otras reflexiones.

\section{MARCO TEÓRICO}

Investigación, educación e innovación son tres propulsores fundamentales y fuertemente interdependientes que apoyan la educación en la Ingeniería, también identificados como "triángulo del conocimiento" [9].

Para la Ingeniería Industrial la integración de los impulsores crea un aprendizaje apoyado en proyectos que buscan soluciones para problemas industriales [9]. Cuando un estudiante de Ingeniería aprende con actividades de Investigación e Innovación se forma con nuevos conocimientos, domina métodos y técnicas para solucionar problemas, experimenta la teoría y práctica, el trabajo en equipo y crea experiencia con situaciones y/o casos reales [9-10].

La internacionalización en la educación superior se considera como un proceso en el marco institucional 
de una universidad. A partir de una revisión de la literatura, se identifica el modelo para la internacionalización de la educación superior, que se presenta en la Figura 1.

Fuente: [11].

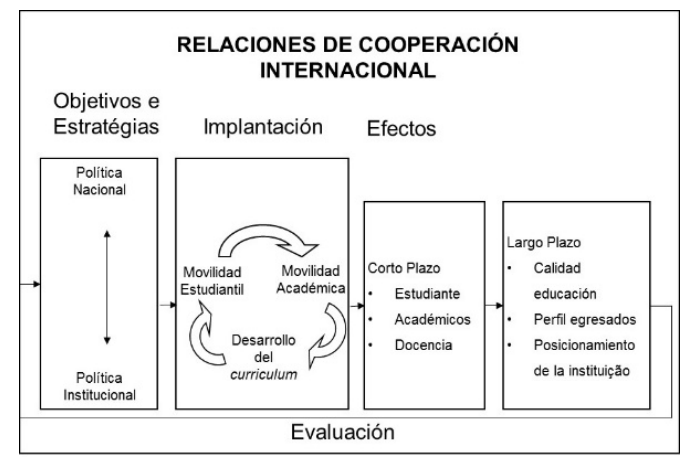

Figura 1. Modelo de internacionalización para la educación superior.

Es un proceso que comienza con la definición de objetivos y estrategias, expresados en documentos de política a nivel gubernamental y a nivel institucional de educación superior.

En la etapa de implantación existen tres elementos centrales: movilidad estudiantil, movilidad de personal académico y desarrollo del curriculum. Los flujos indican la interrelación existente entre ellos. La finalización de este proceso está enfocada en efectos de corto y largo plazo. Los efectos de corto plazo impactan en los estudiantes, los docentes y en las modalidades de enseñanza. Respecto a los efectos de largo plazo, el impacto está reflejado en la internacionalización de la calidad educativa, el perfil de los graduados y el posicionamiento institucional. Existe un lazo de feedback del proceso, que permite su evaluación permanente redefiniendo objetivos y estrategias [11].

Como punto de partida, resulta imprescindible exponer la política educativa nacional respecto a la formación de ingenieros en cada uno de los países participantes de este proceso de cooperación internacional.

Ambos países cuentan con un marco normativo adecuado para el desarrollo de sus carreras de Ingeniería Industrial. En Brasil las Carreras de Ingeniería están reguladas por el Consejo Nacional de Educación (CNE) y la Cámara de Educación
Superior (CE) bajo una sola Resolución, la No 11 de 2002.

En Argentina el Ministerio de Educación, Ciencia y Tecnología junto con el Consejo de Universidades, mediante la Ley $n^{\circ} 24.521$ de Educación Superior establece en su artículo 43 los planes de estudio de carreras correspondientes a profesiones reguladas por el Estado, como las carreras de Ingeniería.

En el ámbito regional, el Sector Educativo del MERCOSUR es un espacio de coordinación de las políticas educativas que reúne los países miembros y asociados del MERCOSUR. A través de la negociación de políticas públicas y la elaboración y ejecución de programas y proyectos conjuntos, el Sector Educativo del MERCOSUR busca la integración y el desarrollo de la educación en toda la región del MERCOSUR y países asociados. Se ha establecido como su misión "Conformar un espacio educativo común, a través de la concertación de políticas que articulen la educación con el proceso de integración del MERCOSUR, estimulando la movilidad, el intercambio y la formación de una identidad y ciudadanía regional, con el objeto de lograr una educación de calidad para todos, con atención especial a los sectores más vulnerables en un proceso de desarrollo con justicia social y respeto a la diversidad cultural de los pueblos de la región." [12].

La síntesis expuesta señala que ambos países han establecido marcos normativos para el desarrollo de la carrera de Ingeniería Industrial, así como un espacio regional de políticas educativas, correspondiendo a un proceso de internacionalización curricular entre diferentes países [13].

\section{FORMULACIÓN DEL MODELO}

Con el fin de implementar estrategias que permitan concretar la internacionalización de la carrera de Ingeniería Industrial entre las universidades, se propone un modelo de cooperación internacional para la integración curricular - $\mathrm{I}^{2} \mathrm{EC}$, según se observa en la Figura 2.

El modelo $I^{2} E C$ es un proceso de internacionalización que busca adquirir conocimientos sobre otros países / región, a partir de un análisis y una comparación de diferentes aspectos culturales, industriales y de 
conocimiento profesional (áreas de conocimiento de la Ingeniería Industrial) dado por las universidades [13].

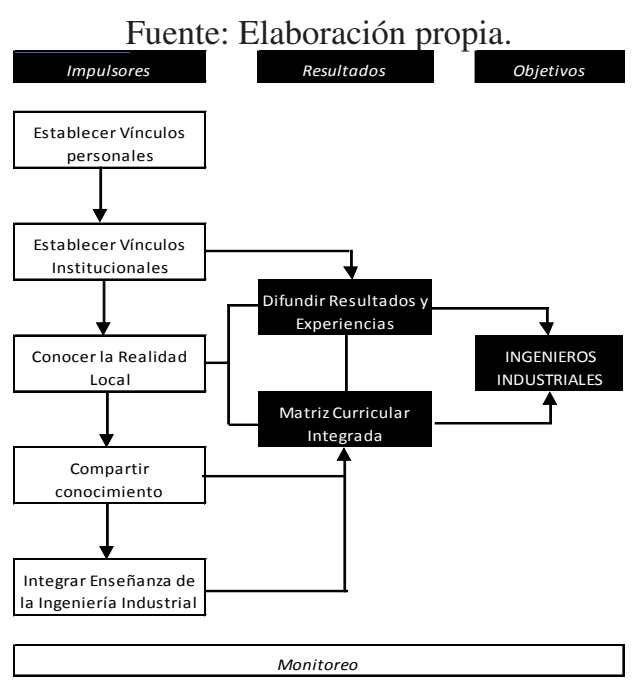

Figura 2. Modelo I²EC.

El modelo conceptual $\mathrm{I}^{2} \mathrm{EC}$ es estimulado por un impulsor, para dar luego inicio a un ciclo iterativo de actividades (representadas por diversas acciones), para lograr un resultado científico y de integración curricular. El objetivo es formar Ingenieros Industriales con dominio de otro idioma, experiencias culturales, industriales y tecnológicas en diferentes regiones, entre otras características profesionales importantes para el egreso.

Cada actividad caracteriza la forma o mecanismos a través de los cuales la cooperación académica en Ingeniería Industrial permite la integración curricular entre universidades de diferentes países. Con cada iteración se adquiere madurez y el sistema se vuelve más robusto.

El punto de partida que da inicio al modelo son vínculos personales preexistentes entre docentes e investigadores de diferentes universidades. En general, esos vínculos son establecidos en reuniones científicas, cursos de graduación o posgraduación, estadías posdoctorales, comisiones científicas, seminarios o workshops, entre otros. Estas relaciones poseen afinidades académicas, científicas y/o profesionales comunes.

A partir de allí, se inicia el ciclo iterativo con una actividad creada para establecer vínculos institucionales. La misma tiene por objetivo formalizar y consolidar en un marco institucional las relaciones personales. Las acciones asociadas a esta actividad son: reuniones con diferentes actores clave de cada una de las instituciones como rectores, responsables de oficinas de cooperación internacional, directores, decanos, buscando lograr el anclaje necesario para el logro de los objetivos propuestos, a través del conocimiento de políticas institucionales y de especificidades de las carreras en cada IES.

Otra acción que debe ejecutarse es la gestión para la formalización de las relaciones interinstitucionales a través de la firma de convenios de cooperación. Actividad que se desarrolla principalmente en el marco de las misiones de trabajo de los profesores a las universidades extranjeras, y se complementan con interacciones periódicas mediante la utilización de las Tecnologías de Información y Comunicación (TIC's).

Conocer la realidad local tiene como finalidad comprender el contexto institucional, social y empresarial de cada una de las universidades. Se proponen como acciones visitas a los campus universitarios, para reconocer su infraestructura y el perfil social de la comunidad estudiantil y visitas técnicas a las empresas donde los estudiantes y graduados se insertan profesionalmente. Las acciones de esta actividad se concentran dentro de las misiones de trabajo.

La actividad propuesta para compartir conocimiento académico tiene como finalidad intercambiar saberes, experiencias y realidades sobre los tres pilares de la misión universitaria (docencia, investigación y extensión). Las acciones relacionadas a ésta son reuniones de trabajo con distintos actores (profesores, coordinadores de carrera) donde se exponen los planes curriculares y se comparten materiales, casos de estudios, esquemas de evaluación, fuentes bibliográficas, entre otros. Además se complementan con visitas a clase para comprender el desarrollo de la docencia en el aula, como las metodologías didácticas empleadas y el abordaje de las temáticas.

Otras acciones pueden corresponder al dictado de charlas y cursos para profesores y estudiantes, en tópicos de interés para la carrera (en este caso, Ingeniería Industrial).

Complementariamente la participación activa en la difusión de las actividades de investigación y 
extensión por parte de la comunidad universitaria permite comprender el interés de los estudiantes en poseer experiencias de movilidad internacional para estudios. Esto pone de relieve el papel central del estudiante en el diseño y ejecución del modelo planteado. Esta actividad, por su diversidad y complejidad, se prolonga a lo largo del tiempo en diferentes formatos, tanto presenciales como virtuales.

Integrar la enseñanza de Ingeniería Industrial es otra de las actividades que conforman este ciclo, cuyo objetivo es idear, planificar y ejecutar prácticas docentes conjuntas entre los profesores que participan de la cooperación internacional y cuyo foco principal son los estudiantes de grado.

Diversas acciones pueden vincularse a esta actividad. A modo de ejemplo, puede enumerarse: elaboración de material didáctico, diseño y dictado de cursos (presencial y/o virtual), integración de perfiles profesionales, análisis de los planes de estudio, identificación de competencias y habilidades, caracterización de áreas de conocimiento comunes de la disciplina, organización de talleres o workshops, etc. Reconociendo a las TIC's como la herramienta imprescindible para llevar adelante esta actividad.

La actividad propuesta para difundir resultados y experiencias tiene como objetivo diseminar los efectos obtenidos hacia la comunidad científica, destacando las fortalezas, buenas prácticas, dificultades y limitaciones en el diseño e implementación del modelo para la integración curricular. En este marco, las publicaciones en eventos académicos, científicos y en revistas especializadas, así como los informes de seguimiento y evaluación constituyen las acciones principales.

Monitoreo es una actividad continua, que involucra todo el proceso de cooperación internacional, permitiendo identificar oportunidades de fortalecimiento así como corregir dificultades y obstáculos de la integración curricular. Se considera a los indicadores como una herramienta valiosa de evaluación que suministra información sobre la implementación del modelo desarrollado [14].

Para concluir con el modelo, teniendo en cuenta el objetivo esperado de la integración curricular, el punto de salida se corresponde al resultado final de una matriz curricular integrada, contemplando un perfil global, nacional y regional del Ingeniero Industrial.

Este modelo es válido de implementar en la medida que exista un compromiso desde las instituciones universitarias, gubernamentales y/o internacionales para dar apoyo político y financiero permitiendo la sustentabilidad de las acciones que componen el modelo propuesto.

\section{IMPLEMENTACIÓN DEL MODELO}

El punto de partida se construyó a partir de una misión de trabajo exploratoria realizada por docentes de UNGS en 2009 hacia la UEM, cuyo propósito fue aproximar un mecanismo de cooperación internacional. A partir de esos esfuerzos y con la participación de distintos investigadores se planeó el proyecto titulado "Cooperación académica para la integración de Ingeniería Industrial en Mercosur" en el marco del Programa Movilidad Docente Mercosur de la SPU. Su objetivo es desarrollar y promover mecanismos de intercambio de conocimiento y experiencias en el área de Ingeniería Industrial.

Del mismo participan tres profesores de la UNICEN, tres profesores de UNGS y dos investigadores de UEM. Se concretaron ocho misiones de trabajo (MT), siete desde Argentina hacia Brasil, y una en sentido inverso. El proyecto se ejecutó desde 2010 hasta 2015, con el financiamiento del Programa y recursos propios de las universidades.

En las Tablas 1, 2, 3, 4, 5 y 6 se exponen las acciones ejecutadas en cada una de las actividades propuestas en el modelo y el periodo de realización de las mismas.

Tabla 1. Actividad: Establecer vínculos institucionales.

\begin{tabular}{|l|l|}
\hline Acciones & Periodo \\
\hline $\begin{array}{l}\text { Reuniones de los profesores argentinos con autoridades } \\
\text { de la universidad y con el coordinador brasilero del } \\
\text { proyecto para idear y planificar actividades conjuntas, } \\
\text { en la visita a UEM. }\end{array}$ & $\begin{array}{l}\text { ler MT } \\
\text { (octubre } \\
2011)\end{array}$ \\
\hline $\begin{array}{l}\text { Los profesores brasileros mantienen reuniones con los } \\
\text { directivos de ambas universidades argentinas, en las } \\
\text { visitas a los sendos campus. }\end{array}$ & $\begin{array}{l}\text { 3er MT } \\
\text { (julio } \\
2013)\end{array}$ \\
$\begin{array}{l}\text { Se inician las gestiones para la firma de convenios } \\
\text { de cooperación académica internacional entre las } \\
\text { universidades. }\end{array}$ & \\
\hline $\begin{array}{l}\text { Se formalizan las TICs como medio de comunicación e } \\
\text { interacción entre los profesores, en forma complementaria } \\
\text { a las misiones de trabajo. }\end{array}$ & Permanente \\
\hline \multicolumn{1}{|c|}{ Fuente: Elaboracion propia. } \\
\hline
\end{tabular}

Fuente: Elaboración propia. 
Tabla 2. Actividad: Conocer la realidad local.

\begin{tabular}{|l|l|}
\hline Acciones & Periodo \\
\hline $\begin{array}{l}\text { Se realizan reconocimientos de los campus } \\
\text { universitarios por parte de los profesores visitantes } \\
\text { (profesores argentinos en UEM y profesores } \\
\text { brasileros en UNGS y UNICEN). }\end{array}$ & $\begin{array}{l}\text { 1er MT (octubre } \\
\text { (julio 2013) }\end{array}$ \\
\hline $\begin{array}{l}\text { Se realizan visitas técnicas a empresas brasileras y } \\
\text { argentinas, para conocer la realidad empresarial. }\end{array}$ & $\begin{array}{l}\text { 1er MT (octubre } \\
\text { 2011) y 3er MT } \\
\text { (julio 2013) }\end{array}$ \\
\hline
\end{tabular}

Fuente: Elaboración propia.

Tabla 3. Actividad: Compartir conocimiento académico.

\begin{tabular}{|l|l|}
\hline Acciones & Periodo \\
\hline $\begin{array}{l}\text { Se realizaron reuniones de intercambio de } \\
\text { conocimiento académico con estudiantes avanzados, } \\
\text { becarios y profesores de la carrera de Ingeniería } \\
\text { Industrial, en las universidades involucradas. }\end{array}$ & $\begin{array}{l}\text { 1er MT (octubre } \\
\text { (juli) 2013) }\end{array}$ \\
\hline $\begin{array}{l}\text { Se realizaron visitas a clase, como forma de } \\
\text { vivenciar la experiencia áulica. }\end{array}$ & $\begin{array}{l}\text { 1er MT (octubre } \\
\text { (jul1) 3er MT }\end{array}$ \\
\hline $\begin{array}{l}\text { Dictado de conferencia "Oportunidades de Intercambio } \\
\text { de Ingeniería Industrial en Mercosur: Argentina". }\end{array}$ & $\begin{array}{l}\text { 1er MT (octubre } \\
\text { 2011) }\end{array}$ \\
\hline $\begin{array}{l}\text { Dictado de conferencia por parte de docentes de } \\
\text { UEM a alumnos de UNICEN y UNGS }\end{array}$ & $\begin{array}{l}\text { 3er MT (julio } \\
\text { 2013) }\end{array}$ \\
\hline $\begin{array}{l}\text { Se dictaron dos conferencias sobre resultados de } \\
\text { proyectos de investigación en el marco del Simposio } \\
\text { Maringaense de Ingeniería de Producción, SIMEPRO. }\end{array}$ & $\begin{array}{l}\text { 2da MT (mayo } \\
\text { (septiembre 2014) }\end{array}$ \\
\hline Dictado de un mini-curso "Revisión Sistémica" (6hs) & 3er MT (julio 2013) \\
\hline
\end{tabular}

Fuente: Elaboración propia.

Tabla 4. Actividad: Integrar enseñanza de Ingeniería Industrial.

\begin{tabular}{|l|l|}
\hline Acciones & Periodo \\
\hline $\begin{array}{l}\text { Se diseñaron las directrices para implementar un curso } \\
\text { virtual a dictar a alumnos de Ingeniería Industrial de las } \\
\text { tres universidades participantes del proyecto. }\end{array}$ & $\begin{array}{l}\text { 2da MT (julio } \\
\text { 2012) }\end{array}$ \\
\hline $\begin{array}{l}\text { Dictado del curso virtual titulado "Intercambio de } \\
\text { conocimiento e integración en Mercosur. Aplicando } \\
\text { herramientas en Ingeniería en Producción en } \\
\text { Pymes argentinas y brasileras" }\end{array}$ & $\begin{array}{l}\text { Octubre 2012 - } \\
\text { abril 2013 }\end{array}$ \\
\hline
\end{tabular}

Fuente: Elaboración propia.

Tabla 5. Actividad: Difundir resultados.

\begin{tabular}{|l|l|}
\hline Acciones & Periodo \\
\hline $\begin{array}{l}\text { Publicación y exposición en Encuentro Nacional de } \\
\text { Ingeniería de Producción(ENEGEP), Salvador, Brasil. [15] }\end{array}$ & $\begin{array}{l}\text { 2da MT } \\
\text { (octubre 2013) }\end{array}$ \\
\hline $\begin{array}{l}\text { Publicación en Congreso en Ingeniería Industrial } \\
\text { (COINI), Mendoza, Argentina. [16] }\end{array}$ & $\begin{array}{l}\text { Noviembre } \\
2013\end{array}$ \\
\hline $\begin{array}{l}\text { Publicación y exposición en Encuentro Nacional de } \\
\text { Ingeniería de Producción (ENEGEP), Curitiba, Brasil. [17] }\end{array}$ & $\begin{array}{l}4 \text { ta MT } \\
\text { (octubre 2014) }\end{array}$ \\
\hline $\begin{array}{l}\text { Publicación en Congreso en Ingeniería Industrial } \\
\text { (COINI), Puerto Madryn, Argentina [18] }\end{array}$ & Octubre 2014 \\
\hline $\begin{array}{l}\text { Publicación y exposición en Encuentro Nacional de } \\
\text { Ingeniería de Producción (ENEGEP), Fortaleza, Brasil. [14] }\end{array}$ & 5ta MT \\
(octubre 2015)
\end{tabular}

Tabla 6. Actividad: Monitoreo.

\begin{tabular}{|l|}
\hline \multicolumn{1}{|c|}{ Acciones } \\
\hline $\begin{array}{l}\text { Seguimiento del proyecto a través de indicadores clave propuestos, } \\
\text { que se agrupan en cuatro categorías: configuración, intensidad, } \\
\text { recursos y resultados [14]. }\end{array}$ \\
\hline
\end{tabular}

Fuente: Elaboración propia.

El punto de salida de este modelo consiste en una matriz curricular integrada, que surge a partir del análisis comparativo de las currículas de Ingeniería Industrial para considerar la compatibilidad académica entre las instituciones. Los resultados del análisis muestran que resultaría factible un programa de intercambio y/o movilidad, en aquellos bloques curriculares que desarrollan las competencias específicas del ingeniero industrial. El análisis académico realizado respecto a la compatibilidad curricular permite viabilizar la movilidad estudiantil entre las instituciones universitarias involucradas, permitiendo desarrollar conocimientos y habilidades al alumno desde el aspecto académico como también experimentar un crecimiento personal por la dimensión socio-cultural [18].

La información evidencia que en una misma misión de trabajo se desarrollaron acciones de diferentes actividades, demostrando el dinamismo y flexibilidad del modelo que se debe poner de manifiesto al llevar adelante el modelo $\mathrm{I}^{2} \mathrm{EC}$.

Concluida la implementación del modelo diseñado, el acople con el modelo teórico de Van der Wende [13], se realiza mediante algunos efectos logrados, en especial aquellos de corto plazo, así como algunos de los de largo plazo que ya se vislumbran. Respecto a los efectos de corto plazo, están enfocados en estudiantes, académicos y la docencia, mientras que los de largo plazo impactan en la calidad educativa e institucional, así como al perfil del graduado.

Los profesores - que han participado de este proceso de internacionalización - compartieron e intercambiaron conocimiento con colegas de otros países, logrando mejorar su práctica docente. Estos docentes adquirieron una visión más amplia del campo académico y profesional vinculado a la carrera de Ingeniería Industrial en este caso. Por último, estas experiencias motivaron a los profesores a continuar y sostener la participación en proyectos de cooperación internacional. Aquellos estudiantes que participaron del curso virtual lograron una interacción internacional, que potencia su formación profesional y su dimensión personal. Como efecto 
indirecto, los estudiantes que recibieron clases con estos profesores recibieron un valor agregado a su formación a partir de la transmisión de las experiencias vividas.

Las prácticas docentes se enriquecieron a partir del intercambio logrado entre los docentes involucrados en el proyecto. El análisis curricular permitió comprender el perfil profesional del ingeniero industrial, así como la compatibilidad de los planes de estudio de cada institución participante, a través de una matriz curricular integrada. Dentro de los efectos de largo plazo se espera lograr una mejora en la calidad educativa, un egresado que logre comprender el fenómeno de lo global y lo regional/ local para insertarse profesionalmente.

Las instituciones involucradas en este proceso de internacionalización logran un posicionamiento institucional diferencial, por ofrecer un valor agregado a su propia comunidad universitaria y a la sociedad en su conjunto.

\section{CONCLUSIONES}

El modelo de Integración Curricular de Ingeniería Industrial (Integration of the Industrial Engineering Curricular $-\mathrm{I}^{2} \mathrm{EC}$ ) fue diseñado e implementado en la carrera de Ingeniería Industrial por las universidades argentinas UNICEN y UNGS, y por la universidad brasilera UEM entre 2010 y 2015, que por sus características permite la replicabilidad para diferentes carreras e instituciones universitarias.

Los resultados obtenidos de su ejecución son considerados satisfactorios para las instituciones integrantes del proyecto. Para los investigadores que participaran fue una oportunidad para intercambiar conocimientos y experiencias de métodos de aprendizaje, proyectos de investigación y disciplinas relacionadas con las carreras de Ingeniería Industrial, asegurando una experiencia internacional y regional de actividades culturales e industriales.

Para los estudiantes fue reforzada la oportunidad de adquirir o aumentar los conocimientos relacionados con la carrera e intercambiar experiencias internacionales, generando un ambiente para discutir conceptos, métodos y herramientas de Ingeniería Industrial aplicados en industrias regionales.
La comparación internacional de los currículos de Ingeniería Industrial demuestra la oportunidad para establecer conocimientos más integrados y cómo algunas diferencias culturales y legislativas pueden ser tratadas y exploradas por actores o responsables por la educación superior en el MERCOSUR.

La integración de más universidades a este proyecto de cooperación internacional le añadiría mayor riqueza universitaria, generando el camino hacia la doble titulación, impulsado por el avance sobre escenarios de educación superior con mayor armonización y homologación académica.

Una investigación futura es determinar cuáles son los efectos e impactos de un currículo integrado en Ingeniería Industrial para estudiantes, docentes, investigadores, universidad e industria regional.

\section{AGRADECIMIENTOS}

Apoyo financiero del Ministerio de Educación de la Nación / Secretaria de Políticas Universitarias de Argentina responsable por el Programa de Asociación Universitaria para Docentes de Grado del Mercosur - Proyecto PMDEM 0052011.

\section{REFERENCIAS}

[1] R. King, S. Marginson and R. Naidoo. "Handbook on Globalization and Higher Education". Edward Elgar Publishing. USA, 2011, pp. 497. ISBN 9781848445857.

[2] H. de Wit, I. C. Jaramillo, J. Gacel-Avila and J. Knight. "Educación superior en América Latina: la dimensión internacional". Banco Mundial / Mayol Ediciones. 2005. pp. 81 ISBN 958-97647-8-9.

[3] D. Crosier, L. Purser and H. Smidt. "Trends V: Universities shaping the European Higher Education Area". European University Association. 2007. ISBN: 9789078997023

[4] M. Larrea y A. Astur. "Políticas de internacionalización de la educación superior y cooperación internacional universitaria". Fecha de consulta: 3 de septiembre 2012. URL: http://portales.educacion.gov.ar/spu/files/2011/12/ Artículo-Políticas-de-internacionalización 
[5] Programa Ciência Sem Fronteiras. Ministério de Educação e Ministério da Ciência, Tecnologia, Inovações e Comunicações. Brasil. Fecha de consulta: 3 de marzo de 2017. URL: http://www.cienciasemfronteiras. gov.br/web/csf/o-programa

[6] http://edu.mercosur.int/arcusur/index.php / es/descripcion/127-sistema-arcu-sur-es

[7] http://educacion.gob.ar/secretaria-depoliticas-universitarias/seccion/44/ sistema-nacional-de-reconocimientoacademico

[8] J. Marangoni Amarante y F. Cortez Verdu. "Um levantamento de publicações sobre internacionalização de instituições de ensino superior". Acta Scientiarum. Human and Social Sciences. Vol. 37 No 2, pp. 127-134. Julio-diciembre. 2015.

[9] G. Chryssolouris, et al. "Manufacturing Systems: Skills \& Competencies for the Futures". Procedia CIRP 7. pp. 17-24. 2013.

[10] C.H. Sturm, et al. "Mapeamento e análise de desempenho da graduação e da pósgraduação em Engenharia de Produção no Brasil”. Gest. Prod., São Carlos. Vol. 22 N $^{\circ}$ 1, pp. 149-163. 2015.

[11] J. Gacel-Avila. "La internacionalización de las universidades mexicanas: políticas y estrategias institucionales". Asociación Nacional de Universidades e Institutos de Educación Superior (México). 2000. ISBN 9687798874.

[12] http://www.edu.mercosur.int/. Recuperada el 4 de Febrero de 2017.

[13] M. van der Wende. "Internationalising the Curriculim in Dutch Higher Education: an International Comparative Perspective"
Journal of Studies in International Education. Vol. $1 \mathrm{~N}^{\circ}$ 53, pp. 53-72. 1997.

[14] F. Chiodi, D. Paravié, E. Cardoza, S. Urrutia y F. Cusolito. "Uso de KPI para autoevaluación de una red de cooperación internacional en ingeniería de industrial / producción". Encontro Nacional de Engenharia de Produção (ENEGEP). Fortaleza, Brasil. Octubre 2015.

[15] E. Cardoza, C. Lapasini Leal, D. Paravié, S. Urrutia y F. Chiodi. "Expandiendo la Formación en Ingeniería Industrial / Producción: Experiencia de Intercambio Virtual entre Estudiantes de Argentina y Brasil" Encontro Nacional de Engenharia de Produção (ENEGEP). Salvador, Brasil. Octubre de 2013.

[16] D. Paravié, S. Urrutia, F. Chiodi, F. Cusolito, C. Lapasini Leal y E. Cardoza. "Experiencia de cooperación internacional para la integración de Ingeniería Industrial". Congreso Argentino de Ingeniería Industrial COINI. Universidad Tecnológica Nacional, Facultad Regional San Rafael. Noviembre de 2013.

[17] V. Cavenaghi, V. Fava de Oliveira y F. Soares Másculo. "Tópicos Emergentes e Desafios Metodológicos em Engenharia de Produção: Casos, Experiências e Proposições". Vol. VIII, ABEPRO - Associação Brasileira de Engenharia de Produção.2016. ISBN: 978-85-88478-49-7.

[18] S. Urrutia, D. Paravié, F. Chiodi, F. Cusolito, C. Lapasini Leal y E. Cardoza. "Hacia la integración curricular de ingeniería industrial en el Mercosur". Congreso Argentino de Ingeniería Industrial COINI, Universidad Tecnológica Nacional, Facultad Regional Chubut. Noviembre de 2014. 